\title{
BUCKLED MEMBRANES FOR MICROSTRUCTURES
}

\author{
Dan S. Popescu' ${ }^{1}$, Theo S. J. Lammerink and Miko Elwenspoek \\ MESA Research Institute, Micro mechanical Transducers Group \\ University of Twente, P.O. Box 217, 7500 AE Enschede, The Netherlands. \\ 1 On leave from: University "Politechnica" Bucharest, Dept. of Electronics, \\ Physical Electronies Group, 77206, Bucharest, Romania.
}

\begin{abstract}
Based on energy variation methods we calculated the deflection of membranes under the combined load of an external pressure and an internal lateral stress. A lateral load gives rise to buckling once a critical load is exceeded. The combination of transversal loads and lateral loads changes the properties of the membrane (and other structures) in the vicinity of the buckling load: The membrane deflects at all lateral loads and the critic load, above which two states are possible shifts. A result important for the design of microsystems, which are based on the buckling phenomenon, is the pressure required to switch the membrane from one state to the other. The theory is tested successfully with micromachined silicon/silicon-dioxide membranes.
\end{abstract}

\section{INTRODUCTION}

The buckling phenomena of thin shells under internal compressive stresses have became very interesting also for micromachined structures. Investigations on pre buckling and post buckling of beams have already been published [1]. Applications of buckling of beams in switches [2], electromechanical memories [3] and applications of buckled membranes in micropumps [4] have been demonstrated. This paper focuses on the mechanism of buckling of the membranes.

The principle of buckling of the membranes is illustrated in Fig. 1.

A membrane under uniform compression $S_{x}=S_{y}=S$, for a compressive stress greater than a critical stress $S_{c r}$, will buckle transversally without any additional external load. There are many specific states of equilibrium with specific patterns of the deflected shape, related to the value of the compressive stress $S$ greater than particular values $S_{c r, \mathrm{i}}$, (i=1...n) with $S_{c r, i+1}>S_{c r, i}$ [5]. In this paper we studied the first order buckling of the membranes. We obtained buckled membranes in a silicon wafer using the compressive stress which exists in a $\mathrm{SiO}_{2}$ thermally grown layer.
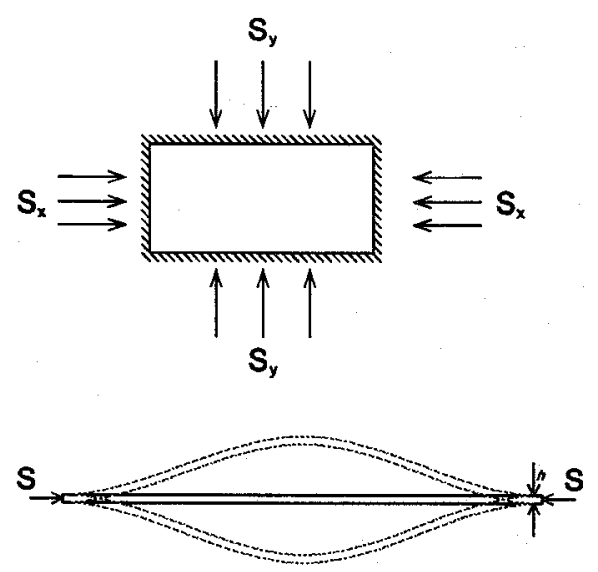

Fig.l Membrane with biaxial plane stress and two stable states for the buckled membrane.

\section{THEORY}

To investigate bending of plates when the stresses are above critical, the strain in the middle plane should be considered. A solution of the problem is to use the expression for the strain energy of the buckled plate and determine its deflection and the critical stress for buckling from the condition that the energy is a minimum.

We made this analysis for the square membrane from Fig.1, with clamped edges and under a uniform compressive stress in two perpendicular directions.

We assume that initially the plate is perfectly flat. The influence of an initial deflection will be taken into consideration later. An expression for the transversal displacement of the buckled plate that satisfies the boundary conditions is:

$w(x, y)=\frac{W_{o}}{4}\left(1+\cos \frac{2 \pi}{a} x\right)\left(1+\cos \frac{2 \pi}{a} y\right)$

where $a$ is the size of the membrane and $W_{o}=w(0,0)$ deflection at the centre of the plate.

The total strain energy of the plate is $U=U_{1}+U_{2}$, with $U_{1}, U_{2}$ the energy of bending and the strain energy due to the forces acting in the middle plane respectively, [6], 
page 386 and page 337 . The relation for the total energy of the plate becomes:

$U=\frac{D W_{o}^{2} \pi^{4}}{a^{2}}+$

$\frac{G h}{1-v} \int_{-a / 2-a / 2}^{a / 2} \int_{x}^{a / 2}\left[e_{x}^{2}+\mathrm{e}_{y}^{2}+2 v e_{x} e_{y}+\frac{1}{2}(1-v) \gamma_{x y}^{2}\right] \mathrm{d} x \mathrm{~d} y$

with

$D=\frac{E h^{3}}{12\left(1-v^{2}\right)}$ and $G=\frac{6 D(1-v)}{h^{3}}$

the flexural rigidity, and the modulus of elasticity in shear respectively. $E$ is Young's modulus, $v$ is Poisson's ratio, $\gamma$ a shear modulus and $h$ is the thickness of the membrane.

From the condition for the energy to be minimum we obtain the uniform compressive stress for first order buckling for a square plate with clamped edges :

$S_{c r}=4.38 \frac{h^{2}}{a^{2}} \frac{E}{1-v^{2}}$

and the expressions for the amplitude of the deflection of the buckled plate under a uniform compressive stress $S$ in two perpendicular directions

$W_{o}=0$, for $S \leq S_{c r}$

$W_{o}= \pm 2.298 h \cdot \sqrt{\frac{S}{S_{c r}}-1}$, for $S \geq S_{c r}$

In the case of a plate with an initial deflection of amplitude $W_{i n}$, the behaviour of the plate under a compressive stress is analysed in the literature for $S<S_{c r}$ and neglecting the work of the forces acting in the middle plane. An approximate expression of the amplitude of the deflection in this case is, [6], page 345:

$W_{o}=\frac{W_{\text {in }}}{1-S / S_{c r}}$ for $S \leq S_{c r}$

This expression is valuable with a good approximation for: $S / S_{c r}<0.6$ [5]. The influence of an initial deflection on the deflection of the buckled plate is shown in Fig. 2.

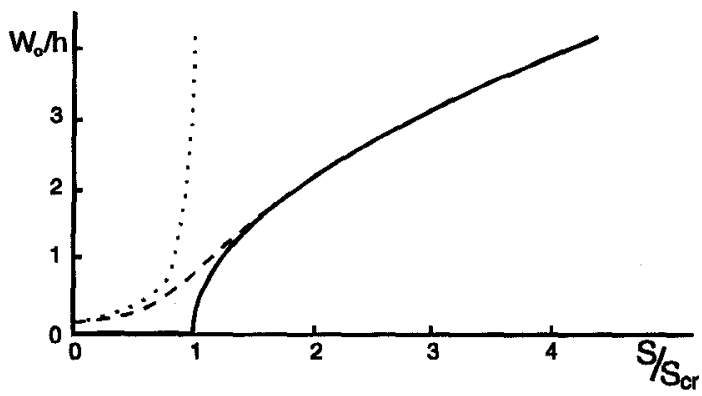

Fig.2 Theoretical curves for the deflection of the rectangular plate under a uniform compressive stress $S$.

The dotted curve represents equation (5) for an initial deflection $W_{\text {in }}=0.1 \mathrm{~h}$. The solid curve represents equation (4) and the dashed curve shows the influence of this initial deflection. The dashed and full curves together show the complete behaviour of the compressed plate.

The total energy of the compressed plate in relation to the plate deflection $W_{o} / h$ and the internal stress $S$ is given by:

$U=33 \frac{D h^{2}}{a^{2}}\left(\frac{W_{o}}{h}\right)^{4}+100 \frac{D h^{2}}{a^{2}}\left(\frac{W_{o}}{h}\right)^{2}\left(1-\frac{S}{S_{c r}}\right)$

and this expression is represented graphically in Fig. 3.

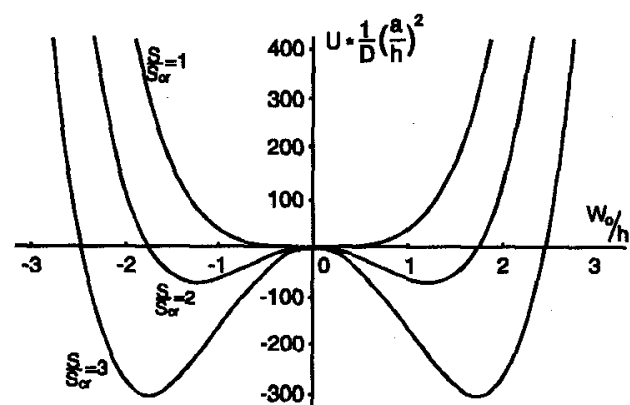

Fig.3 The total strain energy of the plate in relation with the deflection and the internal stress

For $S<S_{c r}$ the energy of the plate has only one minimum for $W_{d} / h=0$.

For $S>S_{c r}$ the energy function has two minima. Both minima indicate a stable state of the stressed membrane.

-external pressure $q$

Now we consider a membrane which is buckled under uniform compressive stress $S>S_{c r}$ and which is loaded with an external pressure $q$. The energy function of the plate is the sum of the energy function given in eq. (6) and the mechanical work by the external pressure: 


$$
\begin{array}{r}
U=33 \frac{D h^{2}}{a^{2}}\left(\frac{W_{o}}{h}\right)^{4}+100 \frac{D h^{2}}{a^{2}}\left(\frac{W_{o}}{h}\right)^{2}\left(1-\frac{S}{S_{c r}}\right)+ \\
q \cdot \frac{a^{2} h}{4}\left(\frac{W_{o}}{h}\right)
\end{array}
$$

Compared to the energy function for a non loaded plate (6) there is an additional term which is linear in $q$ and $W_{o} / h$. Eq. (7) describes the plate energy above and below $S_{c r}$. As an example, the plate energy function $U$ for $S / S_{c r}$ $=3$ with its parametric dependence of the external pressure $q$ is shown in Fig. 4. The energy function is no longer line-symmetric with respect to the y-axis. For relative low external pressures the energy function still does have two minima and two stable states, but for high pressures, there is only one stable minimum left.

In general, the deflection $W_{o}$ for a loaded plate will be a function of both $S$ and $q: W_{o}=W_{o}(S, q)$.

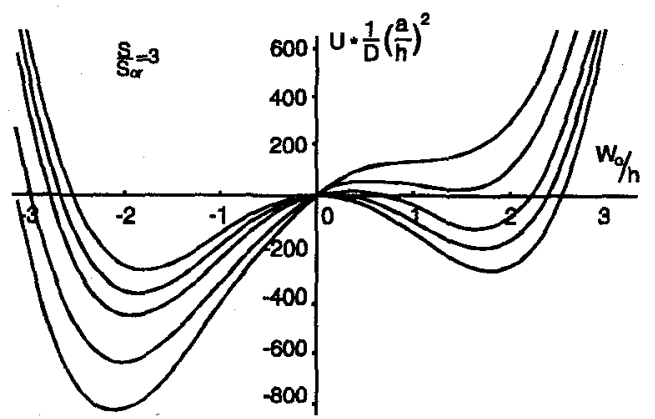

Fig.4. Total strain energy of the plate in relation with the deflection and the external pressure q. The internal stress $S / S_{\mathrm{cr}}=3$.

The minima of the energy function indicate the stable states. The deflection can be calculated from the derivative of the energy function:

$$
\begin{aligned}
\frac{\mathrm{d} U}{\mathrm{~d}\left(W_{o} / h\right)}= & 132 \frac{D h^{2}}{a^{2}}\left(\frac{W_{o}}{h}\right)^{3}+ \\
& 200 \frac{D h^{2}}{a^{2}}\left(\frac{W_{o}}{h}\right)\left(1-\frac{S}{S_{c r}}\right)+q \cdot \frac{a^{2} h}{4}
\end{aligned}
$$

This function is shown in Fig. 5. For a stable state the energy function has a minimum, so this first derivative is zero:

$\mathrm{d} U / \mathrm{d}\left(W_{d} / h\right)=0$

Solving of this equation results in the $q$-dependent deflection: $W_{d} / h(S, q)$. Note that this solution is valid below and above the buckling load. We see that a cubic equation results for the deflection of the membrane as a function of the lateral stress $S$ and the load $q$. This equation has three roots. For three real roots the centre root corresponds to an instable state $\left.\left(\mathrm{d}^{2} U / \mathrm{d}\left(W_{0} / h\right)^{2}\right)<0\right)$.
The two stable roots correspond to the membrane buckled upwards or downwards, respectively. At a certain stress $S_{b}$ (the index $b$ for bifurcation) and pressure $q_{b}$ there are two roots, it is the point where the $S$-shaped curve in Fig. 5 touches the $x$-axis. From this point on going to larger pressures $q$ there is only one stable state (buckled up- or downwards).

The bifurcation point can be looked at from two points of view. One can discuss the deflection as a function of $S$ with $q$ as a parameter. At small $S$ then there is only one real solution. Increasing $S$ the deflection increases, and close to the buckling load, if $q$ is not too large, the deflection increases quite quickly (see the discussion in Söderkvist's paper [10]). At and above the bifurcation point there are two stable solutions, but in practice the deflection will monotonically increase with increasing stress.

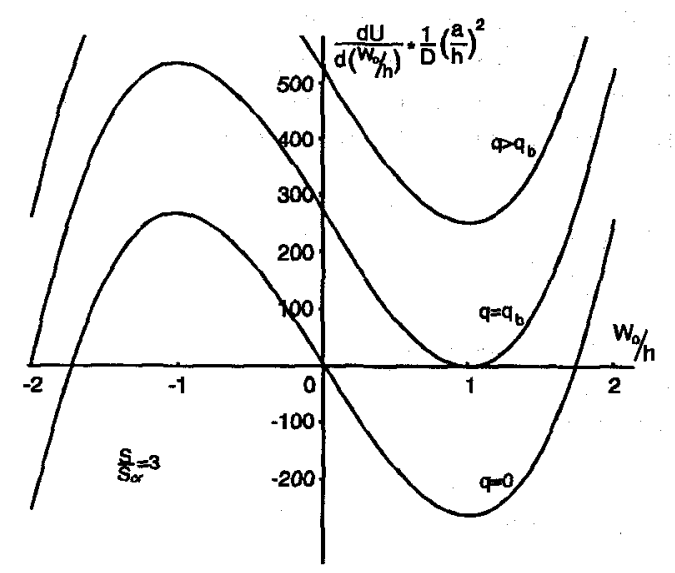

Fig. 5. First derivative of the energy function $U$.

However, if one is in the other branch, and the stress is decreased, the membrane switches to the first state at $S_{b}$. Generally, $S_{b}>S_{c r}$

In fig. 5 we discuss the situation for constant $S$. If $S>S_{c r}$, we have a symmetric situation at $q=0 . q>0$ introduces asymmetry. At constant $S$ the curve in fig. 5 shifts upward and it is seen that the stable deflections shifts to the left: the downward deflection increases and the upward deflection decreases (the sign is arbitrary) until the point is reached where the curve just touches the $\mathrm{x}$-axis. We call this pressure $q_{b}$. At larger pressures there is only one solution. If the membrane is in the upward deflection and the pressure increases, the deflection decreases until a critical pressure $q_{b}$ is reached and the membrane flips to the downward state. This critical pressure is required to switch the membrane. Note that we have a hysteresis; the same argument applies for the downward deflection.

The explicit solution of eq. (9) is standard but quite involved. Therefore we discuss in the following only the 
stiffness of the membrane with respect to the load $q$ at constant stress $S$. We note that a change of $q$ results in a shift along the y-axis of the curve shown in fig. 5 . The following relation therefore is found for the shift of the deflection $\mathrm{d} W_{o}$ by a shift of the load $\mathrm{d} q$ :

$\frac{a^{2} h}{4} \mathrm{~d} q=\frac{\mathrm{d}^{2} U}{\mathrm{~d}\left({ }^{w_{0}} /\right)^{2}} \cdot \mathrm{d}\left(W_{0} / h\right)$

This formula results in the stiffness of the membrane at constant stress

$$
\begin{gathered}
K_{s}=\mathrm{d} W_{o} / \mathrm{d} q=h \cdot \mathrm{d}\left(W_{o} / h\right) / \mathrm{d} q= \\
\frac{a^{2} h}{4}\left(\frac{\mathrm{d}^{2} U}{\mathrm{~d}\left(W_{o} / h\right)^{2}}\right)^{-1}
\end{gathered}
$$

So the effective stiffness of the plate can be calculated from the second derivative of the energy function $U$ :

$$
\begin{aligned}
& \frac{\mathrm{d}^{2} U}{\mathrm{~d}\left(W_{o} / h\right)^{2}}= \\
& \quad 396 \frac{h^{2}}{a^{2}} D\left(\frac{W_{o}}{h}\right)^{2}+200 \frac{h^{2}}{a^{2}} D\left(1-\frac{S}{S_{c r}}\right)
\end{aligned}
$$

For $S>S_{c r}$ we assume that we can make use of eq. (4) for $W_{o}$, so the combination of (10), (11) and (12) results in:

$$
\frac{1}{K_{s}}=6.3 \cdot 10^{2} \frac{h^{3} E^{\prime}}{a^{4}}\left(\frac{S}{S_{c r}}-1\right)
$$

From eq. (13) we see that the effective stiffness is proportional to the internal stress $S$. For $S=S_{c r}$ the effective stiffness for an external pressure $q$ reduces to zero (if $q$ is not too large).

The critical pressure $q_{b}$ is easily calculated by noting that the minimum of the curve labelled $q_{b}$ in Fig. 5 shifts to the $\mathrm{x}$-axis. The position of the minimum is found from eq. (12) and is given by

$$
\frac{W_{o}}{h}=\sqrt{0.51 \cdot\left(1-\frac{S}{S_{c r}}\right)}
$$

$q_{b}$ is calculated from the depth of the minimum,

$$
q_{b}=31.6 \cdot \frac{E^{\prime} h^{4}}{a^{4}}\left(\frac{S}{S_{c r}}-1\right)^{3 / 2}
$$

with $E^{\prime}$ the effective Youngs modulus: $E^{\prime}=E /\left(1-v^{2}\right)$.

\section{EXPERIMENTS}

In our experiment we fabricated buckled membranes in a silicon wafer using the compressive stress, $S_{o}$, which remains in thermally grown $\mathrm{SiO}_{2}$ layer.

From [7] the residual stress $S_{o}$ in thermally grown $\mathrm{SiO}_{2}$ is:

$$
S_{o}=0.3 \cdot 10^{9} \mathrm{~N} / \mathrm{m}^{2}
$$

For a $<100>$ silicon membrane we have [8,9]:

$E^{\prime}=E /\left(1-v^{2}\right)=1.55 \cdot 10^{11} \mathrm{~N} / \mathrm{m}^{2}$

and from equations (3) and (18), we can calculate the thickness of the oxide layer $h_{\mathrm{SiO}_{2}}$ to get the first order buckling:

$$
\begin{aligned}
& h_{\mathrm{SiO}_{2}}=2263 \frac{h^{3}}{a^{2}} \text { for one side } \mathrm{SiO}_{2} \\
& h_{\mathrm{SiO}_{2}}=1132 \frac{h^{3}}{a^{2}} \quad \text { for both sides } \mathrm{SiO}_{2}
\end{aligned}
$$

The relations for $W_{o}$ are than given by:

$$
\begin{aligned}
W_{o}= & 2.298 h \sqrt{\frac{S}{S_{c r}}-1}= \\
& 2.298 h \sqrt{4.419 \cdot 10^{-4} \cdot \frac{h_{\mathrm{SiO}_{2}}}{h} \frac{a^{2}}{h^{2}}-1}
\end{aligned}
$$

Silicon square membranes were anisotropically etched in $\mathrm{KOH}$ and TMAH with mask of thermally grown $\mathrm{SiO}_{2}$ with $1 \mu \mathrm{m}$ thick $\mathrm{SiO}_{2}$ layer at the end of the etching. We studied samples similar to that shown in Fig. 6 with side $a$ between $100 \mu \mathrm{m}$ and $5000 \mu \mathrm{m}$ and thickness $h$ between $12 \mu \mathrm{m}$ and $25 \mu \mathrm{m}$.

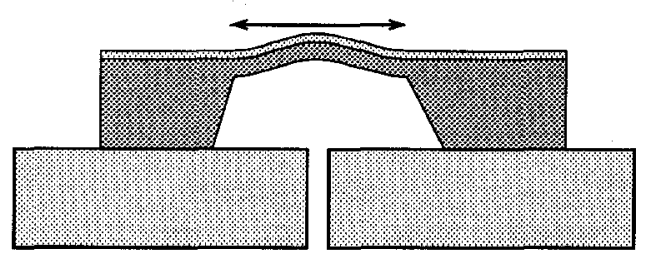

Fig. 6. Cross section of a sample with buckled membrane loaded with a pressure. 


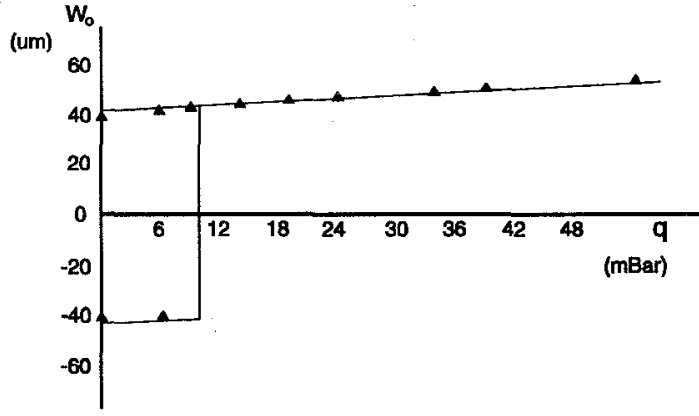

Fig 7. Theoretical and experimental behaviour of the buckled membrane

The membranes buckled into the direction of the $\mathrm{SiO}_{2}$ layer. The membranes buckled according to relation (20) and membranes with $a / h<(a / h)_{c r}$ remained flat. We measured the deflection at the centre of the square membrane, $W_{o}(S, q)$; the pressure difference $q$ was made with a controllable pressure source 'Wallace \& Tiernan' and the deflection was measured with a surface profiler type 'DEKTAK 3030'.

For $\quad h_{\mathrm{SiO}_{2}}=1 \mu \mathrm{m} \quad$ and $\quad a / h=5000 \mu \mathrm{m} / 16 \mu \mathrm{m}, \quad a$ comparison between the theoretical and experimental behaviour of the plate is shown in Fig. 7. Fig.7 shows a linear dependence of $W_{o}$ on $q$.

The theoretically expected buckling pressure is: $q_{b}=11.4$ $10^{2} \mathrm{~N} / \mathrm{m}^{2}$ and the theoretically expected memebrane stiffness is: $\mathrm{d} W_{o} / \mathrm{d} q=4.6 \mu \mathrm{m} / 50 \mathrm{mBar}$. These values agree satisfactory with the experimental results.

A comparison between the pressure required to obtain a particular deflection of buckled and flat membranes is shown in table 1.

\begin{tabular}{|c|c|c|c|}
\hline \multicolumn{4}{|c|}{$a / h=4000 / 18$} \\
\hline deflection $[\mu \mathrm{m}]$ & 21.5 & 28.8 & 37 \\
\hline pressure $^{1}$ [mbar] & 4 & 40 & 100 \\
\hline pressure $^{2}$ [mbar] & 72 & 138 & 255 \\
\hline \multicolumn{4}{|c|}{$a / h=5000 / 16$} \\
\hline deflection $[\mu \mathrm{m}]$ & 42 & 48 & 56 \\
\hline pressure $^{1}$ [mbar] & 6 & 24 & 54 \\
\hline pressure $^{2}$ [mbar] & 105 & 167 & 253 \\
\hline
\end{tabular}

Table 1. Comparison between pressures necessary to deflect buckled and flat membranes.

\section{CONCLUSIONS}

In this work we obtained analytical expressions for the deflection of buckled square membranes under a lateral stress and a pressure difference. The theory also allows for the calculation of the pressure difference needed to switch the membrane from one state to the other. Deflection and switching pressure were determined also experimentally; the results agree well with theory. The main results are:

1) Buckled membranes deflect more than flat membranes under a pressure difference. This fact makes buckled membranes attractive for actuation of micropumps and microvalves.

2) Buckled membranes can be used as memory elements and switched from one state to the other with easily accesable pressures (here: 10 mbar). This fact makes these structures usefull as elements in e.g. two-state valves. The theory presented in this paper makes the design of actuators and memory elements possible.

\section{REFERENCES}

[1] U. Lindberg, J. Söderkvist, T. Lammerink and M. Elwenspoek, "Quasi-Buckling of Micro machined Beams", MME'93, Neuchatel, Switserland, Sept. 1993.

[2] M.A. Huff, A.D. Nikolich and M.A. Schmidt, "A threshold pressure switch utilising plastic deformation of silicon", Proc. Transducers'91, San Francisco, CA, U.S.A., June 1991, 177-180.

[3] B. Hälg, "On a micro-electro-mechanical nonvolatile memory cell", IEEE Tr. Electron. Devices, 37(10) (1990) 2230-2236.

[4] H. Mizoguchi, M. Ando, T. Mizuno, T. Takagi and N. Nakajima, "Design and Fabrication of Light Driven Micropump", IEEE Micro Elecro Mechanical Systems 1992, p. 31-36.

[5] D. Brush, "Buckling of bars, plates and shells", Mc. Graw-Hill (1975).

[6] S. Timoshenko, J. Gere, "Theory of elastical stability", Mc. Graw-Hill N.Y., (1961).

[7] Carl W. Wilmsen, Erik G. Thomson and G.H. Meissner: "Buckling of thermally-grown $\mathrm{SiO} 2$ thin films", IEEE Transactions on Electron Devices Jan. 1972, p.122.

[8] J.J. Wortman, R.A. Evans, "Young's modulus and Poisson's ratio in silicon and germanium", J. Appl. Phys. 36 (1975), p. 113-116.

[9] F.C.M. van de Pol, "A pump based on MicroEngineering Techniques", $\mathrm{PhD}$ thesis, Univ. of Twente, The Netherlands, (1989).

[10]J. Söderkvist and U. Lindberg, "Quasibuckling of Microbridges," in: U. Lindberg, PhD-Thesis, Uppsala University, Sweden, (1993). 